\title{
Sudden and Adiabatic Polarization Effects in Doped Rare Gases
}

\author{
A. M. Köhler, V. Saile, and R. Reininger \\ Hamburger Synchrotronstrahlungslabor HASYLAB, Deutsches Elektronen-Synchrotron DESY, \\ D-2000 Hamburg 52, Federal Republic of Germany \\ and \\ G. L. Findley \\ Department of Chemistry, Louisiana State University, Baton Rouge, Louisiana 70803 \\ (Received 22 February 1988)
}

\begin{abstract}
The evolution of both the sudden and adiabatic polarization energies resulting from a rare gas interacting with a dopant ion was followed for the first time. The variation of these two polarization energies was determined by means of photoabsorption and photoionization up to rare-gas number densities of $(23.0,24.0,11.3$, and 6.6$) \times 10^{20} \mathrm{~cm}^{-3}$ for $\mathrm{He}, \mathrm{Ne}, \mathrm{Ar}$, and $\mathrm{Kr}$, respectively. Both polarization energies were found to vary linearly with rare-gas number density. However, the slopes differ by more than 1 order of magnitude. This difference is explained in terms of a model which accounts for the different time scales of the two experiments.
\end{abstract}

PACS numbers: $33.20 . \mathrm{Ni}, 33.70 . \mathrm{Jg}$

Polarization following electronic excitation is a basic process that occurs in all phases of matter. The effects of this polarization, however, are time-scale dependent. In photoemission from molecular solids, for example, the electron binding energies differ from those of the corresponding free molecule as a result of electronic relaxation during the photoelectron emission process. ${ }^{1}$ This is a fast, or sudden, effect which occurs on a time scale too short to permit intermolecular relaxation. By contrast, an electronic excitation often results also in a geometrical arrangement of the atoms or molecules around the excited center in a solid. As a consequence one observes Stokes shifts for emission lines in fluorescence spectroscopy. This latter example is a slow, or adiabatic, effect. Although the difference between these two polarization energies is intuitively clear its quantitative experimental determination is available only, to our best knowledge, for isolated molecules. ${ }^{2}$ In the present Letter we present, for the same system, the evolution of both polarization energies as a function of the number density.

Our basic idea was to measure the polarization induced by an ion in a rare-gas host. In order to separate the sudden and adiabatic polarization effects, we chose two experimental techniques which differ significantly in their characteristic time scales. In photoabsorption, which has a characteristic time of the order of $10^{-16} \mathrm{~s}$, we measured the energy positions of dopant high- $n$ Rydberg states as a function of rare-gas number density. By extrapolation to the Rydberg series limit, we were able to obtain the density dependence of the vertical ionization energy $\left(I_{v}\right)$ of the dopant. ${ }^{2}$ Beyond a certain host density, however, a dopant high- $n$ Rydberg state can polarize the medium to such an extent that relaxation to an electron-ion pair becomes energetically possible. The time scale of this process is given by the lifetime of the
Rydberg state involved $\left(10^{-12} \mathrm{~s}\right)$. In order to detect the adiabatic energy onset $\left(I_{\mathrm{ad}}\right)$ of electron-ion pair production, we simultaneously measured photoionization spectra as a function of host number density. In this way, we were able to follow the evolution of both the sudden $\left(I_{v}\right)$ and adiabatic $\left(I_{\text {ad }}\right)$ polarization energies as a function of host number density up to the highest number densities ever studied in this type of experiment.

The model system chosen for this study was methyl iodide $\left(\mathrm{CH}_{3} \mathrm{I}\right)$ doped into varying number densities of $\mathrm{He}, \mathrm{Ne}, \mathrm{Ar}$, and $\mathrm{Kr}$. We should emphasize, however, that the general results reported here are characteristic of the host rather than of the dopant.

The details of the experimental arrangement used in the measurements reported here are described elsewhere. ${ }^{3}$ Briefly, monochromatic synchrotron radiation was focused into the center of a 10-mm-long experimental cell which was equipped with entrance and exit $\mathrm{LiF}$ windows, and with a pair of parallel-plate electrodes oriented perpendicular to the windows. A highresolution monochromator ${ }^{4}$ permitted the precise (resolution $\approx 2 \mathrm{meV}$ ) determination of Rydberg-state excitation energies and photoionization threshold energies. In all cases, the concentration of the dopant was less than $10 \mathrm{ppm}$.

In Fig. 1, typical photoionization spectra, and one absorption spectrum, are compared for the system $\mathrm{CH}_{3} \mathrm{I}$ Ar. The photoionization onset, ${ }^{5}$ which clearly shifts to lower photon energies as the host number density increases, is indicated in each spectrum.

The density dependence of the absorption spectra of $\mathrm{CH}_{3} \mathrm{I}$ doped into $\mathrm{He}, \mathrm{Ne}, \mathrm{Ar}$, and $\mathrm{Kr}$ has been reported. ${ }^{6}$ Our main finding was that the energy shift of high- $n$ Rydberg states, and consequently of the series limit, $I_{v}$, is a linear function of the host number density for all 


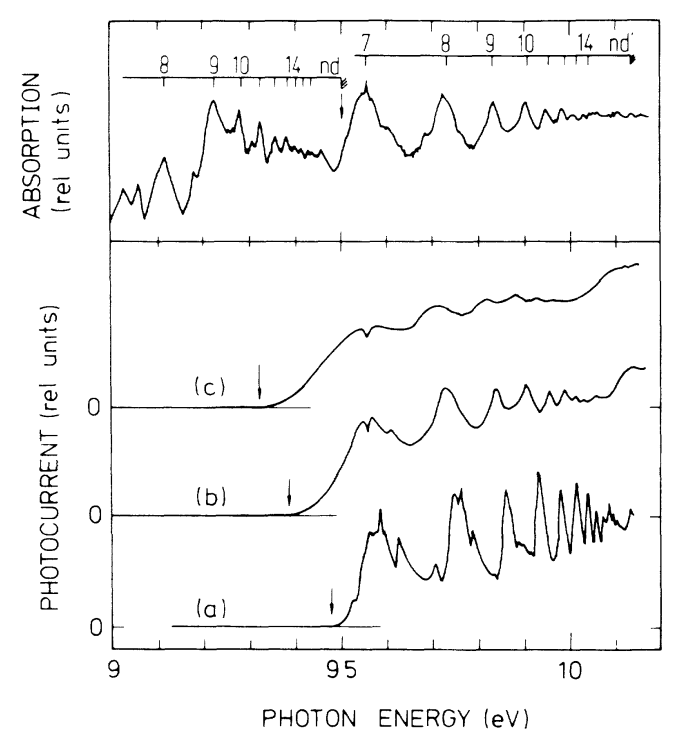

FIG. 1. Bottom: Photoionization spectra of $\mathrm{CH}_{3} \mathrm{I}$ in $\mathrm{Ar}$ : (a) pure $\mathrm{CH}_{3} \mathrm{I}$ at $p=0.1 \mathrm{Torr}$; (b),(c) the Ar number density is $5.7 \times 10^{20} \mathrm{~cm}^{-3}$ and $11.3 \times 10^{20} \mathrm{~cm}^{-3}$, respectively; the $\mathrm{CH}_{3} \mathrm{I}$ concentration is less than $10 \mathrm{ppm}$. The arrows indicate the onset of the photocurrent. Top: Absorption spectrum recorded simultaneously with the photoionization spectrum in (b). The assignment of the shifted $n d$ and $n d^{\prime}$ Rydberg series is given. The arrow indicates the position of the limit of the series converging to the first ionization limit.

four rare gases. The Rydberg states broaden with number density and could be followed up to densities of $(23.0,24.0,11.3$, and 6.6$) \times 10^{20} \mathrm{~cm}^{-3}$ for $\mathrm{He}, \mathrm{Ne}, \mathrm{Ar}$, and $\mathrm{Kr}$, respectively. The linear shift observed for the whole density range was discussed ${ }^{6}$ in terms of a Fermi model. $^{7}$ The energy shift $\Delta$ is the sum of two contributions, namely,

$$
\Delta=\Delta_{\mathrm{sc}}+\Delta_{\mathrm{pol}}
$$

where $\Delta_{\mathrm{sc}}$ is the contribution due to the scattering of the quasi free electron off of the perturbing atoms, and $\Delta_{\mathrm{pol}}$ is the sudden polarization of the medium by the ion core.

The electron-scattering shift is given by ${ }^{7}$

$$
\Delta_{\mathrm{sc}}=\left(2 \pi h^{2} / m\right) a \rho,
$$

where $m$ is the mass of the optical electron, $a$ is the electron-rare-gas scattering length, and $\rho$ is the host number density.

For the polarization contribution to Rydberg-state energy shifts, Fermi suggested ${ }^{7}$

$$
\Delta_{\mathrm{pol}}=-\left(\alpha e^{2} 4 \pi \rho / 2\right) \int_{R_{0}}^{\infty} R^{-4} R^{2} d R,
$$

where $\alpha$ is the rare-gas atomic polarizability, $R$ is the ion-rare-gas separation, and $R_{0}$ is a finite lower integration limit. In the original Fermi model, ${ }^{7} R_{0}$ was taken to be the Wigner-Seitz radius: $R_{0}=(3 / 4 \pi \rho)^{1 / 3}$. In our

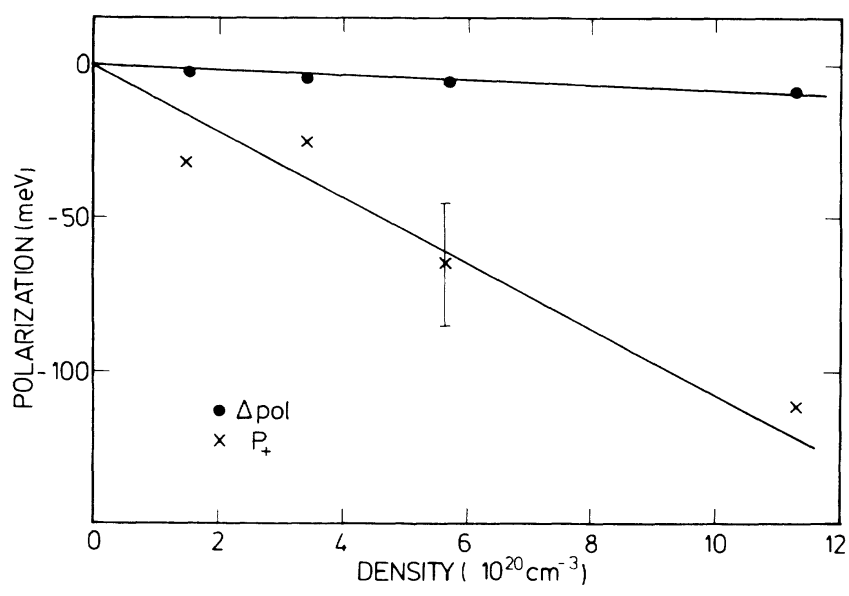

FIG. 2. Sudden and adiabatic polarization energies of the methyl iodide ion as a function of Ar number density. Circles, experimental values of $\Delta_{\mathrm{pol}}$. (The experimental error is as small as the diameter of the points.) Crosses, experimental values of $P_{+}$. (The experimental error is shown for one point.) The lines represent, in both cases, the calculated polarization energies. See text for details.

earlier work, ${ }^{6}$ however, we demonstrated that the Wigner-Seitz radius is an inappropriate choice for the lower integration limit. If we introduce the Weisskopf radius, $R_{\mathrm{W}}=\left(\pi \alpha e^{2} / 4 h v\right)^{1 / 3}$ (where $v$ is the thermal velocity of the rare-gas atoms), as the lower integration limit in Eq. (3), we find

$$
\Delta_{\mathrm{pol}}=-10.78\left(\alpha e^{2 / 2}\right)^{2 / 3}(h v)^{1 / 3} \rho .
$$

The introduction of the Weisskopf radius in Eq. (3) is justified by the fact that we measure the energy shift of the line center. And, this shift is determined only by host atoms situated outside of the Weisskopf sphere. ${ }^{8}$ By contrast, atoms within the Weisskopf sphere contribute to line broadening rather than to the shift of the line center.

Equation (4) is practically identical to the expression obtained by Alekseev and Sobel'man ${ }^{8,9}$ for the very lowdensity case. Moreover, Eq. (4) describes our previous experimental results quantitatively, ${ }^{6}$ as shown by the upper line in Fig. 2. Our experimental results are summarized and compared with theory [Eqs. (2) and (4)] in Table I.

In the density range present here, we may express the shift in the adiabatic ionization energy as

$$
\Delta I_{\mathrm{ad}}=\Delta_{\mathrm{sc}}+P_{+},
$$

where $P_{+}$is the adiabatic polarization energy. The experimental density dependence of $P_{+}$is presented in Fig. 2 for the case $\mathrm{CH}_{3} \mathrm{I}-\mathrm{Ar}$. Note that $P+$ is strikingly larger than $\Delta_{\mathrm{pol}}$. This is also borne out for the other rare gases, as shown in Table I, although the results for $\mathrm{He}$ and $\mathrm{Ne}$ are less accurate.

As discussed above, $\Delta_{\text {pol }}$ is determined only by those 
TABLE I. Experimental (expt) shifts of the vertical $(\Delta)$ and adiabatic $\left(\Delta I_{\text {ad }}\right)$ ionization energies with number density $\rho$. The scattering $\left(\Delta_{\mathrm{sc}}\right)$ and polarization $\left(\Delta_{\mathrm{pol}}, P_{+}\right)$contributions to the shifts were calculated (calc) with Eqs. (2), (4), and (6), respectively. The experimental values for $\Delta_{\mathrm{pol}}$ and $P+$ were obtained from Eqs. (1) and (5). All these quantities are given in units of $10^{-23} \mathrm{eV} \mathrm{cm}^{3}$. The lower part of the table pertains to the parameters for the model potentials [Eq. (7)] employed to calculate $P_{+}$.

\begin{tabular}{lcccc}
\hline \hline & $\mathrm{Kr}$ & $\mathrm{Ar}$ & $\mathrm{Ne}$ & $\mathrm{He}$ \\
\hline$\Delta / \rho($ expt $)$ & $-8.6 \pm 0.2$ & $-5.1 \pm 0.4$ & $0.09 \pm 0.03$ & $2.44 \pm 0.06$ \\
$\Delta I_{\mathrm{ad}} / \rho$ (expt) & $-36.4 \pm 4.8$ & $-14.4 \pm 2.1$ & $-2.4 \pm 1.5$ & $-0.4 \pm 0.7$ \\
$\Delta_{\mathrm{sc}} / \rho$ (calc) & -8.11 & -4.31 & 0.61 & 2.92 \\
$\Delta_{\mathrm{pol}} / \rho(\mathrm{expt})$ & $-0.5 \pm 0.2$ & $-0.8 \pm 0.4$ & $-0.52 \pm 0.03$ & $-0.48 \pm 0.06$ \\
$\Delta_{\mathrm{pol}} / \rho(\mathrm{calc})$ & -0.84 & -0.73 & -0.31 & -0.27 \\
$P+/ \rho(\mathrm{expt})$ & $-28.3 \pm 4.8$ & $-10.1 \pm 2.1$ & $-3.0 \pm 1.5$ & $-3.3 \pm 0.7$ \\
$P+/ \rho(\mathrm{calc})$ & -28.5 & -10.1 & -2.7 & -1.2 \\
$E(\mathrm{meV})$ & $18.39^{\mathrm{a}}$ & $14.30^{\mathrm{a}}$ & $2.85^{\mathrm{a}}$ & $1.03^{\mathrm{a}}$ \\
$\sigma(\AA)$ & 5.1 & 4.7 & 4.6 & 4.4 \\
$\bar{\alpha}$ & $13.772^{\mathrm{b}}$ & $13.772^{\mathrm{b}}$ & $13.772^{\mathrm{b}}$ & $13.722^{\mathrm{b}}$ \\
$F(\mathrm{meV})$ & $385^{\mathrm{a}}$ & $176^{\mathrm{a}}$ & $41^{\mathrm{a}}$ & $20^{\mathrm{c}}$ \\
$R_{m}(\AA)$ & 3.7 & 3.6 & 3.5 & 3.2 \\
$\beta(\AA-1)$ & $1.7^{\mathrm{d}}$ & $1.7^{\mathrm{d}}$ & $1.7^{\mathrm{d}}$ & $1.7^{\mathrm{d}}$ \\
\hline \hline
\end{tabular}

${ }^{\mathrm{a}}$ Reference 10 .

${ }^{\mathrm{b}}$ For $\bar{\alpha}=13.772$ the modified Buckingham potential can be smoothly connected to a Lennard-Jones potential given by $E, \sigma$.

${ }^{c}$ Estimated.

${ }^{\mathrm{d}}$ Reference 11 .

perturbers outside of the Weisskopf sphere. $P_{+}$, however, is determined predominantly by those perturbers within the Weisskopf sphere, as we will show below.

In order to calculate the contribution of close perturbers, we must consider the molecular potential curves that describe the interaction between the dopant in the ground state and the perturber, $V$, and that between the ion core and the perturber, $V_{+} . P+$ is then given by

$$
P_{+}=4 \pi \rho \int_{0}^{\infty}\left(V-V_{+}\right) W(R) R^{2} d R,
$$

where $W(R)$ describes the distribution of perturbers around the ion core.

In order to model our experimental data, we chose a modified Buckingham potential for $V$, and a Morse potential for $V_{+}$:

$$
\begin{aligned}
& V=\frac{E}{1-6 / \bar{\alpha}}\left\{\frac{6}{\bar{\alpha}} \exp \left[-\bar{\alpha}\left(1-\frac{R}{\sigma}\right)\right]-\left(\frac{\sigma}{R}\right)^{6}\right\}, \\
& V_{+}=F\left\{1-\exp \left[-\beta\left(R-R_{m}\right)\right]\right\}^{2}-F .
\end{aligned}
$$

In the low-density range that we consider here, $W(R)$ is given by the classical expression:

$$
W(R)=\exp (-V / k T) \text {. }
$$

For the case of $\mathrm{CH}_{3} \mathrm{I}-\mathrm{Ar}, W(R)$ is shown in Fig. 3, along with $V$ and $V+$. Since, to our knowledge, the parameters for the molecular potentials are now known, and since $\mathrm{CH}_{3} \mathrm{I}$ and $\mathrm{Xe}$ exhibit many similarities in their electronic

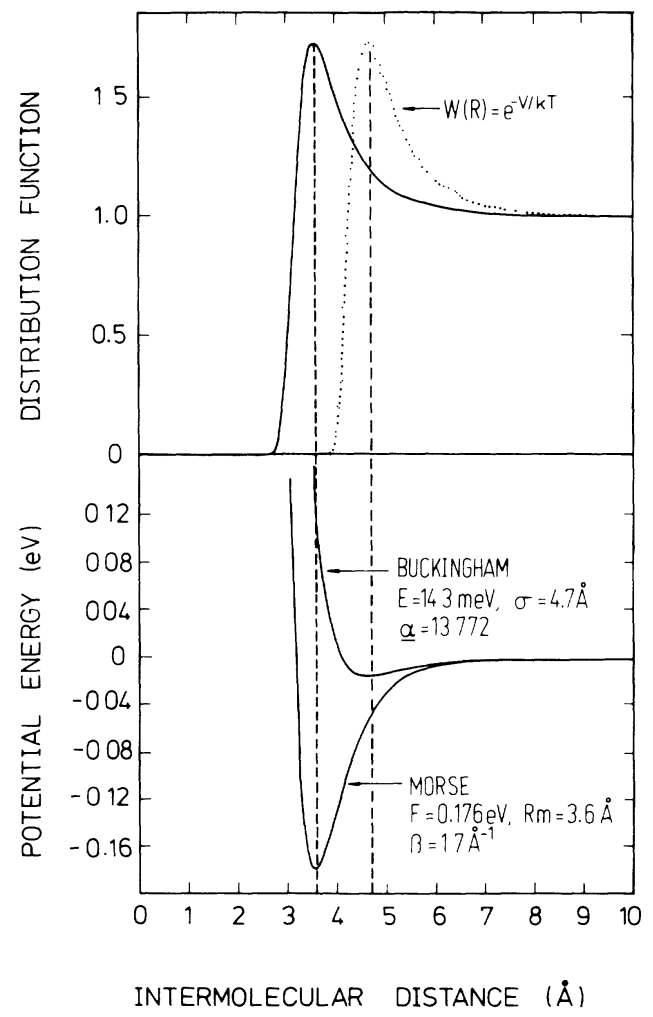

FIG. 3. Bottom: Model potential curves, given by Eq. (7). Top: distribution function calculated from Eq. (8) (dotted line), and shifted to $R=R_{m}$ (full line). 
structure, $^{12}$ we chose parameters pertaining to $\mathrm{Xe}$ Ar. ${ }^{10,11}$

In order to calculate $P_{+}$, we must first consider the evolution of $W(R)$ in time. Before and during the photoabsorption process, $W(R)$ is determined by the ground-state potential $V$. Subsequent to the excitation to a Rydberg state, perturbers begin to move in the direction of the dopant core, under the influence of $V_{+}$. This we model by a rigid translation of $W(R)$ (cf. Fig. 3). This rigid translation is justified by the fact that the relaxation process is limited by the lifetime of the Rydberg state, which is of the order of $10^{-13}-10^{-12} \mathrm{~s}$ (as estimated from linewidths in photoabsorption). Thus, there is insufficient time for a full molecular relaxation.

Under the above assumptions, we calculated $P+$ as a function of host number density. The result for $\mathrm{CH}_{3} \mathrm{I}-\mathrm{Ar}$ is given by the lower line in Fig. 2. Clearly, the agreement between theory and experiment is excellent. The results for the other rare gases are summarized in Table I. Finally, it is worth pointing out that these model calculations are mainly sensitive to the depth of $V_{+}$, and to the relative distance $\sigma-R_{m}$.

In summary, we have presented new experimental data which permit us to separate the sudden and adiabatic polarization effects in doped rare gases, and followed their evolution as a function of medium density. In addition, we have provided model calculations which reproduce quantitatively our experimental results. We wish to point out, however, that at very high densities $\rho>5 \times 10^{21} \mathrm{~cm}^{-3}$ repulsive forces must be taken into account. In this case, $P+$ and $\Delta_{\text {sc }}$ are no longer linear in $\rho .^{13}$

This work was supported in part by the U.S. Department of Energy.

${ }^{1}$ H. Inokuchi, K. Seki, and N. Sato, Phys. Scr. T17 93
(1987).

${ }^{2}$ See, e.g., K. Kimura, S. Katsumata, Y. Achiba, T. Yamazaki, and S. Iwata, Handbook of HeI Photoelectron Spectra of Fundamental Organic Molecules (Halsted, New York, 1981).

${ }^{3}$ V. Saile, R. Reininger, A. M. Köhler, and G. L. Findley, Nucl. Instrum. Methods Phys. Res., Sect. A238, 558 (1985); R. Reininger, I. T. Steinberger, S. Bernstorff, V. Saile, and P. Laporte, Chem. Phys. 86, 189 (1984).

${ }^{4}$ V. Saile, P. Gürtler, E. E. Koch, A. Kozevnikov, M. Skibowski, and W. Steinmann, Appl. Opt. 15, 2559 (1976).

${ }^{5}$ Adiabatic photoionization onsets were determined by our normalizing all photoionization spectra to the same photocurrent, and assigning as the onset that energy for which an initial rise in photocurrent was detected. It is important to note, however, that the host-induced shift in onset reported here is independent of the specific method chosen to determine the onsets.

${ }^{6}$ A. M. Köhler, R. Reininger, V. Saile, and G. L. Findley, Phys. Rev. A 33, 771 (1986), and 35, 79 (1987); A. M. Köhler, Ph.D. thesis, University of Hamburg, 1987 (unpublished).

${ }^{7}$ E. Fermi, Nuovo Cimento 11, 157 (1934); see also H. S. W. Massey and E. H. S. Burshop, Electronic and Ionic Impact Phenomena (Oxford Univ. Press, London, 1952), p. 178.

${ }^{8}$ V. A. Alekseev and I. I. Sobel'man, Zh. Eksp. Teor. Fiz. 49, 1274 (1965) [Sov. Phys. JETP 22, 882 (1966)].

${ }^{9}$ Only the numerical prefactors differ slightly: Alekseev and Sobel'man report -9.87 compared to -10.78 in Eq. (4).

${ }^{10}$ P. M. Dehmer and S. T. Pratt, in Photophysics and Photochemistry in the Vacuum Ultraviolet, edited by $\mathrm{S}$. P. McGlynn, G. L. Findley, and R. H. Huebner, NATO Advanced Study Institute Series C, Vol. 142 (Reidel, Dordrecht, Holland, 1984).

${ }^{11}$ I. Y. Fugol, Phys. 27, 1 (1978).

${ }^{12}$ H. T. Wang, W. S. Felps, G. L. Findley, A. R. P. Rau, and S. P. McGlynn, J. Chem. Phys. 67, 3940 (1977).

${ }^{13}$ R. Reininger, U. Asaf, and I. T. Steinberger, Chem. Phys. Lett. 90, 287 (1982); R. Reininger, U. Asaf, I. T. Steinberger, and G. Basak, Phys. Rev. B 28, 4426 (1983); R. Reininger, V. Saile, P. Laporte, and I. T. Steinberger, Chem. Phys. 89, 473 (1984). 